\title{
Generalized pustular psoriasis
}

INSERM

\section{Source}

INSERM. (1999). Orphanet: an online rare disease and orphan drug data base.

Generalized pustular psoriasis. ORPHA:247353

Generalized pustular psoriasis is a severe inflammatory skin disease that can be lifethreatening and that is characterized by recurrent episodes of high fever, fatigue, episodic erythematous cutaneous eruptions with sterile cutaneous pustules formation on various parts of the body, and neutrophil leukocytosis. 